\title{
Low Power CMOS Chopper Preamplifier Based on Source-Degeneration Transconductors
}

\author{
O. J. Cinco-Izquierdo - M.T. Sanz-Pascual \\ Electronics Department \\ Instituto Nacional de Astrofísica, \\ Óptica y Electrónica (INAOE) \\ Tonantzintla, Puebla, México \\ Email: \{oscar.cinco, materesa $\} @$ inaoep.mx
}

\author{
C. A. de la Cruz-Blas \\ Department of Electrical \\ and Electronic Engineering \\ Universidad Pública de Navarra \\ Pamplona, Spain \\ Email: \{carlos.aristoteles\}@unavarra.es
}

\author{
B. Calvo-López \\ Group of Electronic Design (I3A) \\ Universidad de Zaragoza \\ Zaragoza, Spain \\ Email: $\{$ becalvo $\} @$ unizar.es
}

\begin{abstract}
This paper describes the design of a low-power, low noise CMOS chopper preamplifier for sensor signal conditioning. The core amplifier and the Gm-C output low pass filter of the proposed fully differential preamplifier are based on a source degeneration transconductor. The circuit was designed in a standard $0.18 \mu \mathrm{m} C M O S$ process with $1.8 \mathrm{~V}$ supply voltage. It shows $42 d B$ gain, $1 \mathrm{kHz}$ bandwidth and a total power consumption of $84 \mu \mathrm{W}$. The proposed configuration achieves a noise efficiency factor of 4.6 and a total input-referred noise of $560 \mathrm{n} V_{r m s}$ integrated from 0.1 to $1 \mathrm{kHz}$.
\end{abstract}

Index Terms-Low-noise Preamplifier, Chopping Technique, Analog Front-End.

\section{INTRODUCTION}

Preamplifiers are fundamental building blocks in sensor signal conditioning, as they primarily determine the performance of the whole acquisition system. They are required to amplify, with a well-defined gain, very weak differential signals, in the order of $m V$ or $\mu V$, with minimum power consumption in order to comply with portable, wearable and implantable application requirements [1-2]. High commonmode rejection ratio (CMRR) and high power supply rejection ratio (PSRR) are also necessary to attenuate environmental interference. Furthermore, many sensors provide output signals near DC with a few $\mathrm{Hz}$ bandwidth, especially in biosignal acquisition systems [3-6], so flicker noise is the main source of intrinsic noise. In order to reduce the low frequency noise, dynamic offset cancellation techniques are used, which can be classified into two groups: auto-zero and chopping [7]. The auto-zero technique consists in first sampling and then subtracting the offset and low frequency noise of the amplifier, but undersampling of the broadband noise results in an increased thermal noise contribution. The chopping technique, in turn, is a continuous time modulation technique in which the signal is translated to higher frequency, amplified and demodulated back to baseband, whereas the flicker noise is only modulated once and then filtered. As there is no noise undersampling, the residual noise is lower than with the auto-zero technique. In this paper a fully differential low power chopper preamplifier is presented. The core amplifier is based on a source-

This work has been supported by CONACYT Doctoral Grant 467255 and CONACYT CB-2015-01-257985 Research Project. degenerated transconductor to provide a well-defined gain and higher bandwidth than open-loop operational transconductance amplifiers, thus avoiding phase shift between the input and output modulators. The output low-pass filter is based on the same transconductor with reduced power consumption, so a low noise efficiency factor (NEF) is achieved for the whole preamplifier. The paper is organized as follows. Section II presents the block diagram of the proposed system and some design considerations. The core amplifier and its characteristics are described in Section III, and the low power first-order $G m-C$ filter is presented in Section IV. Simulation results and comparison with previous low noise preamplifiers are provided in Section V. Finally, some conclusions are drawn in Section VI.

\section{PRoposed PREAMPLIFIER}

Figure 1 shows the block diagram of the proposed preamplifier. It is designed to process input signals from $5 \mu \mathrm{V}$ to $1 \mathrm{mV}$ with frequencies from $0.1 \mathrm{~Hz}$ to $250 \mathrm{~Hz}$. The core amplifier (CA) must provide a gain about $40 d B$ or higher, to ensure that it determines the overall noise of the acquisition system, and must be carefully designed to reduce its intrinsic noise contribution. The chopper technique is applied to the CA by means of an external chopper modulator $\mathrm{CH} 1$, an internal chopper modulator $\mathrm{CH} 2$ embedded in the output stage of the $\mathrm{CA}$, and a $G m-C$ low-pass filter (LPF).

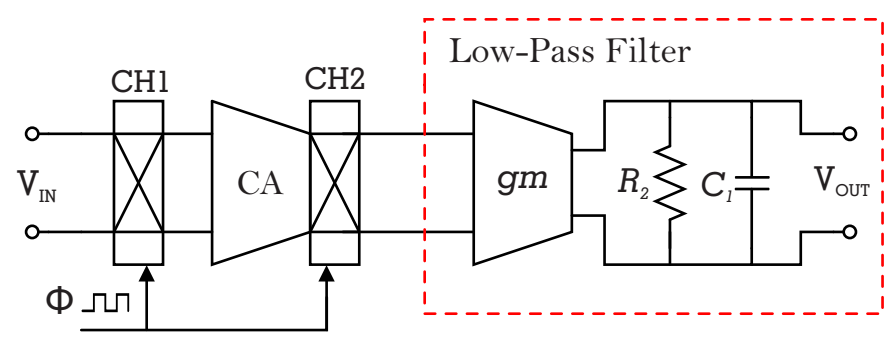

Fig. 1. Proposed Low-Noise System.

The input modulator $C H 1$ consists of four transmission gates to reduce charge injection [7], whereas the output modulator $\mathrm{CH} 2$ makes use of the low impedance nodes generated 
by cascode transistors, as shown in the next Section, thus reducing glitches and resulting in a more compact solution. The chopper modulators are controlled by a square wave signal with frequency $f_{\text {chop }}=100 \mathrm{kHz}$. Finally, the LPF is based on the same transconductor cell as the $\mathrm{CA}$, with reduced power consumption and a cut-off frequency of $1 \mathrm{kHz}$ to eliminate the modulated noise components.

\section{CORE AMPlifier (CA)}

The CA consists of a flipped-voltage follower (FVF) based source degenerated transconductor, as shown in Figure 2. The DC current through the input transistors $M_{1}$ and $M_{2}$ is held constant which, together with the low impedance node established at their source terminals, results in unity voltage gain and high current sourcing capability [8]. The output current through $M_{5}$ and $M_{6}$ is therefore determined by the differential input voltage and the degeneration resistor $R_{S}$. The output current is copied through $M_{7}$ and $M_{8}$ and converted into a differential output voltage by means of resistor $R_{L}$, so the gain of the CA is $M \cdot R_{L} / R_{S}$, where $M$ is the gain of the current mirror. Cascode transistors $M_{S W}$ in the output branches constitute the output modulator $\mathrm{CH} 2$, whereas $M_{3}$ and $M_{4}$ were added to increase the accuracy in the copy of currents. The common mode feedback (CMFB) circuit, not shown in the Figure, consists of a differential difference amplifier [9].

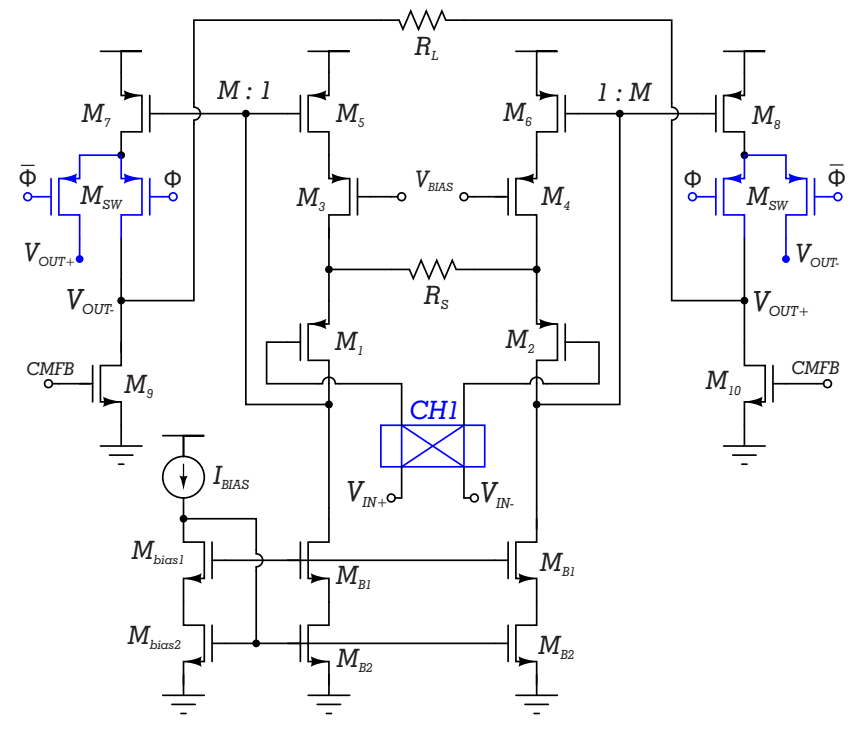

Fig. 2. Core Amplifier.

The input-referred flicker noise of the CA is given by:

$$
\begin{aligned}
V_{n, 1 / f}^{2} & =\frac{2}{C_{o x} f}\left[\frac{K_{p}}{(W L)_{1}}+g_{m 5}^{2} R_{S}^{2}\left[\frac{K_{p}}{(W L)_{5}}+\frac{K_{p}}{\left(W L_{7}\right)}\right]\right] \\
& +\frac{2}{C_{o x} f} \cdot \frac{g_{m B 2}^{2}\left(1+g_{m 1} R_{S}\right)^{2}}{g_{m 1}^{2}} \cdot \frac{K_{n}}{(W L)_{B 2}} \\
& +\frac{2}{C_{o x} f} \cdot \frac{g_{m 9}^{2}}{g_{m 7}^{2}} \cdot g_{m 5}^{2} R_{S}^{2} \cdot \frac{K_{n}}{(W L)_{9}}
\end{aligned}
$$

where $K_{n}$ and $K_{p}$ are technology dependent constants, $C_{o x}$ is the oxide capacitance, $f$ is the frequency, and $g_{m i}$ and $(W L)_{i}$ are the transconductance and area of transistor $M_{i}$, respectively. As $R_{S}$ and $R_{L}$ are linear polysilicon resistors, they only contribute with thermal noise, which is not taken into account in eq. (1). If transistors $M_{7}-M_{8}$ are designed $M$ times wider than $M_{5}-M_{6}$ to provide some gain to the current mirror, their area and transconductance are also $M$ times higher. Therefore, assuming $g_{m 1} R_{S}>>1$, eq. (1) can be rewritten as:

$$
\begin{aligned}
V_{n, 1 / f}^{2} & =\frac{2 \cdot K_{p}}{C_{o x} f}\left[\frac{1}{(W L)_{1}}+\frac{g_{m 5}^{2} R_{S}^{2}(1+M)}{M(W L)_{5}}\right] \\
& +\frac{2 \cdot K_{n}}{C_{o x} f}\left[\frac{g_{m B 2}^{2} R_{S}^{2}}{(W L)_{B 2}}+\frac{g_{m 9}^{2} R_{S}^{2}}{M^{2}(W L)_{9}}\right]
\end{aligned}
$$

The gain $M$ of the current mirror should be chosen higher than 1 to reduce flicker noise. However, there is a trade-off between the reduction in flicker noise and the increase in power consumption due to the increase in the current through the output branches. For this reason, $M=1.5$ was chosen. Also, as $K_{p}$ is lower than $K_{n}$, it is preferable to use a PMOS FVF input, and in order to obtain the highest power efficiency, and thus the best noise-power trade-off, large area transistors operating in the weak inversion region are used. Table I summarizes the sizes of the transistors. The bias current of the amplifier is $I_{b i a s}=5 \mu \mathrm{A}$, the degeneration resistance $R_{S}=1 k \Omega$, and the output resistance $R_{L}=100 \mathrm{k} \Omega$. The main characteristics of the CA are shown in Table II. The amplifier shows an input-referred noise of $9.7 \mu V_{r m s}$, integrated in the noise-bandwidth (from 0.1 to $880 \mathrm{kHz}$ ), and $70 \mu \mathrm{W}$ power consumption. The input-referred noise integrated from 0.1 to $1 k H z$, which will be the integration range after applying the chopping technique to the $\mathrm{CA}$, is $1 \mu V_{r m s}$.

TABLE I

TRANSISTOR SIZES

\begin{tabular}{|c|c|c|}
\hline & $W(\mu m)$ & $L(\mu m)$ \\
\hline \hline$M_{1,2}$ & 528 & 1 \\
\hline$M_{3,4}$ & 28 & 1 \\
\hline$M_{5,6}$ & 88 & 1 \\
\hline$M_{7,8}$ & 132 & 1 \\
\hline$M_{9,10}$ & 352 & 1 \\
\hline$M_{B 1}$ & 112 & 1 \\
\hline$M_{B 2}$ & 352 & 1 \\
\hline \hline
\end{tabular}

TABLE II

Characteristics OF THE CORE AMPLIFIER

\begin{tabular}{|c|c|}
\hline & Core Amplifier \\
\hline \hline Gain & $40 \mathrm{~dB}$ \\
\hline Bandwidth & $560 \mathrm{kHz}$ \\
\hline Power & $70 \mu W$ \\
\hline CMRR & $74 \mathrm{~dB} @ 250 \mathrm{~Hz}$ \\
\hline PSRR & $70 \mathrm{~dB} @ 250 \mathrm{~Hz}$ \\
\hline THD @ $1 m V_{p p}$ & $0.5 \% @ 250 \mathrm{~Hz}$ \\
\hline Input-Referred Noise & $9.7 \mu V_{r m s}$ \\
\hline
\end{tabular}




\section{Filter Design}

Figure 3 shows the first-order fully differential Gm-C filter used as the output LPF in the chopping technique. Although it is based on the same FVF transconductance topology as the CA, it was redesigned to contribute less than $20 \%$ to the power consumption of the whole preamplifier. This implies higher noise, but as the filter is preceded by the low noise high gain $\mathrm{CA}$, its noise contribution to the proposed preamplifier is still negligible.

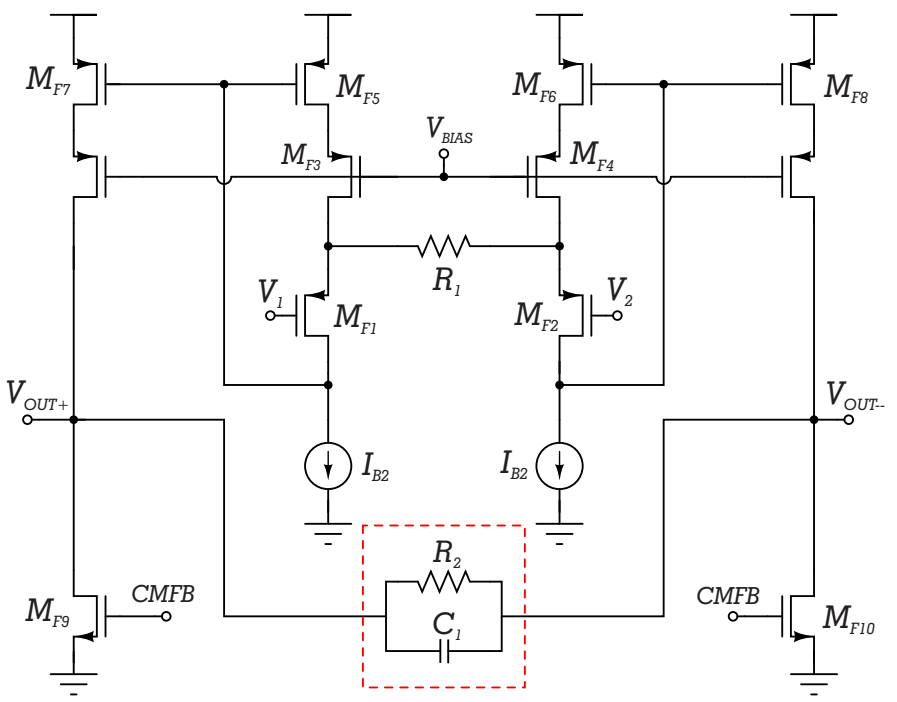

Fig. 3. First-order Gm-C filter.

The transfer function of the filter is given by:

$$
H(s)=\frac{R_{2}}{R_{1}\left(1+s C_{1} R_{2}\right)}
$$

The cut-off frequency was chosen $f_{c}=1 \mathrm{kHz}$ to filter out the noise components modulated by $\mathrm{CH} 2$ and process input signals up to $250 \mathrm{~Hz}$. To achieve such a low cutoff frequency, large capacitive and resistive values are required, which implies large area consumption if passive components are used. For this reason, resistors $R_{1}$ and $R_{2}$ were implemented as pseudo-resistors, as shown in Figure 4. The PMOS transistors are biased in the weak inversion region with bulk-drain or bulk-source terminals shorted. This pseudo-resistor exhibits a weak dependence on $V_{12}$, which results in a large resistance with moderate linearity for the input voltage ranges of the system [10].

In this way, a resistance $R_{2}=4.5 M \Omega$ is achieved, which only takes $0.52 \mu \mathrm{m}^{2}$ active area, as each transistor size is $W / L=0.36 \mu \mathrm{m} / 0.36 \mu \mathrm{m}$. To establish the $1 \mathrm{kHz}$ cut-off frequency, a capacitance $C_{1}=35 \mathrm{pF}$ is required. The DC gain of the filter is 2 , providing some additional gain to the preamplifier. Finally, the power consumption of the proposed Gm-C filter is $14 \mu \mathrm{W}$ and its input-referred noise is $6 \mu V_{r m s}$.

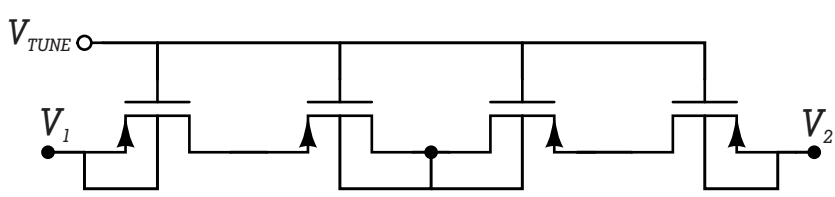

Fig. 4. Pseudo-Resistor Implementation.

\section{Simulation Results}

The proposed circuit was designed in a $0.18 \mu \mathrm{m}$ CMOS process with $1.8 \mathrm{~V}$ supply voltage, and consumes $84 \mu \mathrm{W}$ total power. The preamplifier frequency response is shown in Figure 5. The circuit presents a differential gain of $42 \mathrm{~dB}$ and $1 \mathrm{kHz}$ bandwidth. Figure 6 shows the time response for a $500 \mu \mathrm{V}$ amplitude sine input signal at $250 \mathrm{~Hz}$. The demodulated and amplified input signal is shown before filtering the modulated noise (Fig. 6a), and the output of the LPF (Fig. 6b). The total harmonic distortion under these conditions is $1.5 \%$.

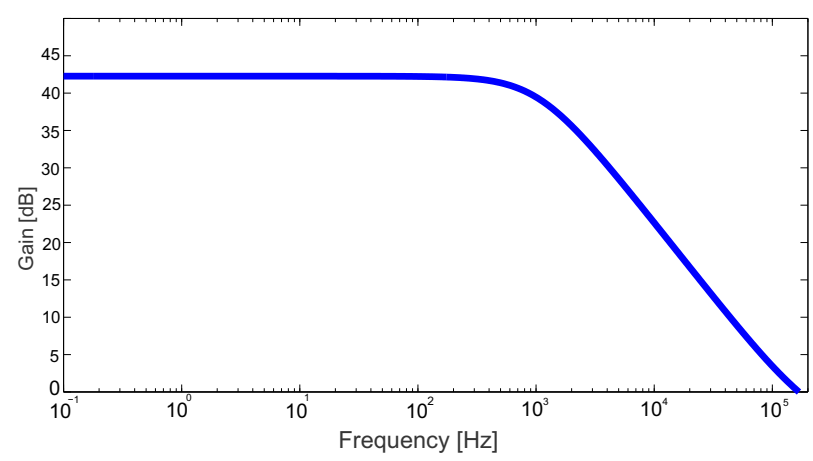

Fig. 5. Preamplifier frequency response.

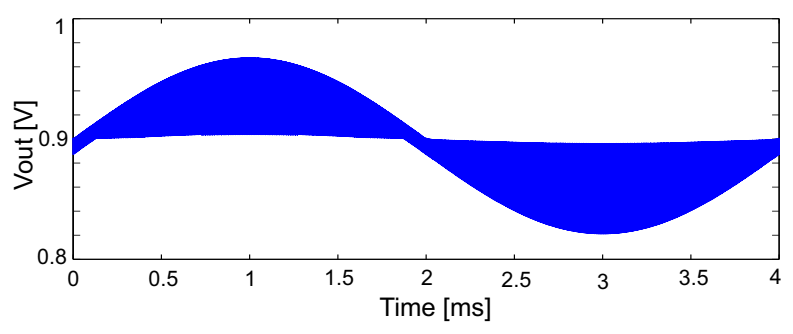

(a)

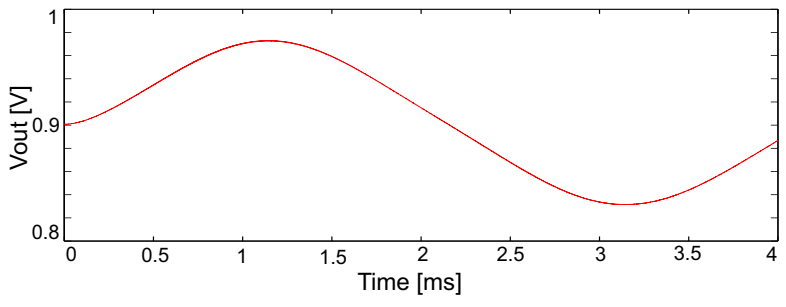

(b)

Fig. 6. Waveforms at the a) input and b) output of the LPF. 
The trade-off between the input-referred noise and the current consumption of the amplifier is usually expressed in terms of the noise efficiency factor $(N E F)$ [11]:

$$
N E F=V_{n i, r m s} \cdot \sqrt{\frac{2 \cdot I_{\text {total }}}{\pi \cdot 4 k T \cdot V_{t} \cdot B W}}
$$

where $V_{n i, r m s}$ is the input-referred noise voltage integrated in the bandwidth of the preamplifier $B W, I_{\text {total }}$ is the current consumption, $V_{t}$ is the thermal voltage, $k$ is the Boltzmann constant and $T$ is the temperature. Another figure of merit used to compare the design of amplifiers operating with different supply voltages is the power efficiency factor $(P E F)$, defined as $N E F^{2} \cdot V_{D D}$. The smaller the $P E F$, the better the trade-off between noise and power consumption.

The equivalent input-referred noise power spectral density of the core amplifier and the proposed chopper preamplifier is shown in Figure 7. At $250 \mathrm{~Hz}$ the input-referred noise decreases from $24.5 \mathrm{nV} / \sqrt{\mathrm{Hz}}$ without the chopping technique, to $14.5 \mathrm{nV} / \sqrt{\mathrm{Hz}}$ with the chopping technique. When integrated from $0.1 \mathrm{~Hz}$ to $1 \mathrm{kHz}$, the input-referred noise is $1 \mu V_{r m s}$ for the $\mathrm{CA}$, and $0.56 \mu V_{r m s}$ for the whole configuration. The efficiency factors of the proposed preamplifier are $N E F=4.6$ and $P E F=38 \mathrm{~V}$.

Table III shows a comparison with previous implementations found in the literature. Although reference [5] achieves the lowest NEF and PEF, no information about linearity is provided. As shown in the table, the proposed preamplifier is very competitive, as it provides an excellent trade-off between all the relevant parameters, with low NEF and PEF, high CMRR and PSRR and moderate linearity.

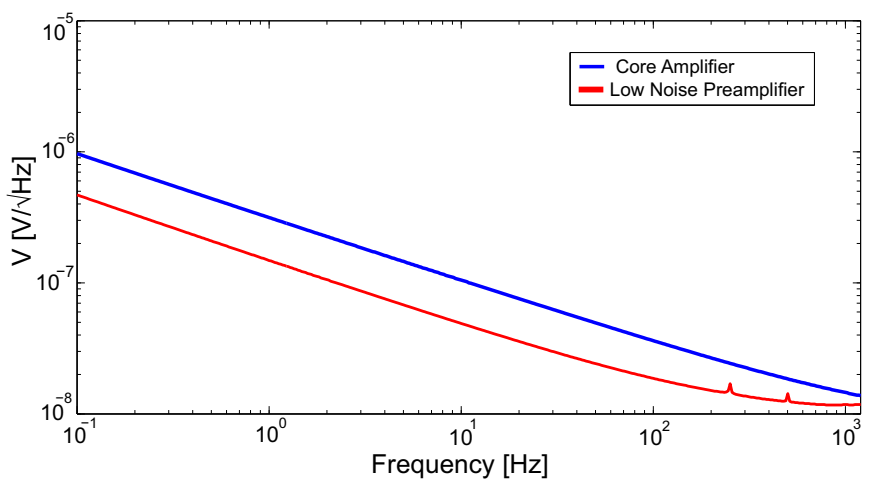

Fig. 7. Square root of the input-referred power spectral density.

\section{CONClusions}

A low power low noise preamplifier designed in a $0.18 \mu \mathrm{m}$ CMOS process with $1.8 \mathrm{~V}$ supply was presented in this paper. It uses a source degeneration transconductor as building block to design the core amplifier (CA) and the $\mathrm{Gm}-\mathrm{C}$ output filter. A careful design process was undertaken to achieve the best noise-power trade-off in the $\mathrm{CA}$ and thus achieve the best efficiency factors after applying the chopping technique. The proposed preamplifier shows an input-referred noise of
TABLE III

PERformance COMPARISON OF PREAMPLIFIERS

\begin{tabular}{|c|c|c|c|c|}
\hline & {$[4]$} & {$[5]$} & {$[6]$} & This Work \\
\hline \hline Technology $(\mu m)$ & 0.18 & 0.18 & 0.13 & 0.18 \\
\hline Power Supply $(V)$ & 1.8 & 1.2 & 1 & 1.8 \\
\hline Gain $(d B)$ & $48 / 60$ & $40-54$ & 55 & 42 \\
\hline Bandwidth $(H z)$ & $1-8000$ & $1-100$ & $0.01-100$ & $0.1-1000$ \\
\hline Power $(\mu W)$ & 11 & 0.8 & 2.3 & 84 \\
\hline CMRR $(d B)$ & 48 & 105 & 125 & $74 @ 250 \mathrm{~Hz}$ \\
\hline PSRR $(d B)$ & 55 & - & - & $70 @ 250 \mathrm{~Hz}$ \\
\hline THD $(\%)$ & $\begin{array}{c}1.2 \\
1 \mathrm{~m} V\end{array}$ & - & - & $\begin{array}{c}1.5 \\
\text { ( } 1 \mathrm{mV}\end{array}$ \\
\hline $\begin{array}{c}\text { Input-Referred } \\
\text { Noise }\left(\mu V_{r m s}\right)\end{array}$ & 5 & 1 & 0.75 & 0.56 \\
\hline NEF & 5.3 & 3.3 & 4.5 & 4.6 \\
\hline PEF $(V)$ & 50 & 13 & 20 & 38 \\
\hline \hline
\end{tabular}

$0.56 \mu V_{r m s}$ in an integration bandwidth of $0.1 \mathrm{~Hz}$ to $1 \mathrm{kHz}$, $1.5 \%$ THD for a $1 m V_{p p}$ input signal at $250 \mathrm{~Hz}$, and power consumption of $84 \mu \mathrm{W}$. The efficiency factors are $N E F=4.6$ and $P E F=38 \mathrm{~V}$.

\section{ACKNOWLEDGMENT}

This work was supported by CONACYT through the Doctoral Grant 467255 and the Research Project CONACYT CB2015-257985.

\section{REFERENCES}

[1] S. Ma and Y. Cheng, "Circuit Design Challenges and Considerations for Portable Medical Instrument System Applications," 14th IEEE International Conference on Solid-State and Integrated Circuit Technology (ICSICT), Qingdao, 2018, pp. 1-4.

[2] H. Bhamra, J. Lynch, M. Ward and P. Irazoqui, "A Noise-Power-Area Optimized Biosensing Front End for Wireless Body Sensor Nodes and Medical Implantable Devices," in IEEE Transactions on Very Large Scale Integration (VLSI) Systems, vol. 25, no. 10, pp. 2917-2928, Oct. 2017.

[3] M. Maslik, T. S. Lande and T. G. Constandinou, "A Clockless Method of Flicker Noise Suppression in Continuous-Time Acquisition of Biosignals," IEEE Biomedical Circuits and Systems Conference (BioCAS), Cleveland, $\mathrm{OH}, 2018$, pp. 1-4.

[4] P. Kmon and P. Gryboś, "Energy Efficient Low-Noise Multichannel Neural Amplifier in Submicron CMOS Process", in IEEE Transactions on Circuits and Systems I: Regular Papers, vol. 60, no. 7, pp. 1764-1775, July 2013.

[5] Liang, Zhiming et al. "A Fully Integrated Chopper IA for Implantable Multichannel EEG Recording Without Impedance Boosting Circuits.' IEEE Asia Pacific Conference on Circuits and Systems (APCCAS) 2018, pp: 143-146.

[6] C. Lee and J. Song, ”A Chopper Stabilized Current-Feedback Instrumentation Amplifier for EEG Acquisition Applications," in IEEE Access, vol. 7, pp. 11565-11569, 2019.

[7] C. C. Enz and G. C. Temes, "Circuit techniques for reducing the effects of op-amp imperfections: autozeroing, correlated double sampling, and chopper stabilization," in Proceedings of the IEEE, vol. 84, no. 11, pp. 1584-1614, Nov. 1996

[8] R. G. Carvajal et al., "The flipped voltage follower: a useful cell for lowvoltage low-power circuit design," in IEEE Transactions on Circuits and Systems I: Regular Papers, vol. 52, no. 7, pp. 1276-1291, July 2005.

[9] D. A. Johns and K. Martin "Analog Integrated Circuit Design", John Wiley and Sons, 2012.

[10] D. Palomeque-Mangut, J. L. Ausín, F. Duque-Carrillo and G. Torelli, "Design of robust pseudo-resistors with optimized frequency response,' 2017 European Conference on Circuit Theory and Design (ECCTD), Catania, 2017, pp. 1-4.

[11] M. S. J. Steyaert and W. M. C. Sansen, "A micropower low-noise monolithic instrumentation amplifier for medical purposes", in IEEE Journal of Solid-State Circuits, vol. 22, no. 6, pp. 1163-1168, Dec. 1987. 